\title{
Effects of Kav001 (A Kavain Analogue) on LPS-Induced Cytokines and Neutrophil Infiltration
}

\author{
Xiaoren Tang ${ }^{1 *}$, Mansour Alasiri ${ }^{1}$, Yinian Tang ${ }^{2}$, Feng $\mathrm{Cao}^{1}$ and Bing $\mathrm{Liu}^{3}$ \\ ${ }^{1}$ Department of Periodontology, Boston University Goldman School of Dental Medicine, USA
}

${ }^{2}$ Boston University School of Medicine, USA

${ }^{3}$ Department of General Dentistry, Boston University Goldman School of Dental Medicine, USA

*Corresponding author: Xiaoren Tang, Department of Periodontology, Boston University Goldman School of Dental Medicine, 650 Albany Street, Boston, MA 02118, USA

\begin{abstract}
Background: TNF- $\alpha$, a cytokine, is known to be involved in LPS-induced inflammation. Our recent data indicate that treatment of Kavain in mouse or human cells significantly suppresses TNF- $\alpha$ production in response to LPS. In order to completely identify one Kavain analogue (Kav001) reported in our previous data, we further analyzed its biological function in this paper.

Methods and Results: Commercial Kavain or Kav001 as inhibitor was used. ELISA was performed for detection of TNF- $\alpha$, IL-1 $\beta$, IL-6, IL-4, or Caspase 1 after treatment of THP-1 cells with Kav001. Wild type mice (C57BL/6) were used for neutrophil infiltration assay. A kinase/protein array was performed to analyze if Kav001 affects any kinases/protein in response to LPS. Conclusions: It is clear that Kav001 not only inhibits TNF- $\alpha$, but also IL-1 $\beta$, IL-6, caspase 1 and neutrophil infiltration in response to LPS compared to control.
\end{abstract}

\section{Introduction}

Kavain is known as a treatment for certain diseases [1-3]. Amar's group recently reported that, in vivo, Kavain mediated alveolar bone loss and inflammatory infiltrate in a mnouse model [4]. Kavain is found to affect TNF- $\alpha$ production through regulation of NF-kB [5] and to be inhibited by ERK2 dephosphorylation in vitro or in vivo [6]. Our previous data indicate that Kavain inhibits $\mathrm{P}$. gingivalis/LPS-induced inflammation/inflammatory disease [7,8]. Recently, we found that Kavain grants small amounts of toxicity to mammalian cells, most likely due to its insoluble chemical structure with hydrophobicity. To address this issue, we designed and synthesized compounds with the basic chemical structure of Kavain with a different number of hydrophilic bonds. One soluble compound (named Kav001) from the Kavain analogue was selected and further confirmed to have a stronger biological function than Kavain by in vitro/in vivo experiments such as CAIA mouse models or endotoxic shock [9]. In this paper, we further found that Kav001 not only inhibits TNF- $\alpha$, but also IL-1 $\beta$, IL-6, caspase 1 and neutrophil infiltration. However, this phenomenon cannot be observed when macrophages are treated with LPS plus Kavain. We believe that Kav001 may mediate a novel link between Kav001 and LPS-induced inflammation and may be used as a key inhibitor to LPS-induced inflammation/inflammatory disease.

\section{Materials and Methods \\ Animals and cells}

Wild type mice (8-10 week-old C57BL/6, Jackson Labs) were maintained under strict specific pathogen-free (SPF) conditions. All protocols were approved by the Boston University Institutional Animal Care and Use Committee and were performed in compliance with the relevant animal care and use laws and institutional guidelines. THP-1 cells (TIB-202, ATCC) were cultured in a RPMI1640 media (Cat\#: 11875-093, Life Technologies, NY) with $10 \%$ FBS at $37^{\circ} \mathrm{C}$ in a $5 \% \mathrm{CO} 2$ atmosphere.

\section{Compounds}

Commercial Kavain (Cat\#59780, Sigma-Aldrich Co., Natick, MA) and custom synthesized analogue Kav001 (Lot\#KB180125, KareBay Biochem, Inc.) were obtained. The analysis of each 
compound shows that $\geq 95 \%$ purity. The stock concentration of each compound was prepared with $10 \mu \mathrm{g} / \mu \mathrm{l}$. The compound was diluted to $100 \mu \mathrm{g} / \mathrm{ml}$ in $25 \%$ DMSO for Kavain or in water for Kav001 just before the experiment.

\section{Elisa}

The treated media from THP-1 cells were subjected to ELISA for TNF- $\alpha$ detection with the kit (Cat\#: ab181421, Abcam), IL-1 $\beta$ detection with the kit (Cat\#: ab100562, Abcam), IL-6 detection with the kit (Cat\#: KHC0061, Life technologies), IL-4 detection with the kit (Cat\#: BMS225-2, Thermo Fisher), or Caspase 1 (Cat\#: ab219633, Abcam). ELISA immunoreactivity was measured by a reader (Model 680, Bio-Rad). The data were graphed.

\section{Neutrophil Infiltration Assay}

Mice ( $n=3$ ) were untreated or treated by i.p. injection of E.coli LPS (1mg/kg body weight) and/or oral gavage of Kav001 (30mg/ $\mathrm{kg}$ ) for $24 \mathrm{hrs}$. Skin tissues from each group were taken and fixed in $4 \%$ paraformaldehyde, then embedded in O.C.T. compound $[10,11]$. Skin sections $(5 \mu \mathrm{m})$ were made and stained with H\&E (hematoxylin and eosin). Results were analyzed and imaged.

\section{Protein Array}

$1 \times 105$ THP- 1 cells were treated with $100 \mu \mathrm{g} / \mathrm{ml}$ of E. coli LPS (Sigma) or plus Kav001 $\left(0.1 \mathrm{mg} / \mathrm{ml}\right.$ ) for $16 \mathrm{hrs}$ at $37^{\circ} \mathrm{C}$. The medium from each test was centrifuged at 1,500 $\times \mathrm{g}$ and then treated with a specific kit for human protein cytokine array (RayBiotech, Norcross, GA) based on the manufacturer's instructions. The data were analyzed using a Verrado Imaging System (Bio-Rad) and graphed.

\section{Statistical Analysis}

All tests were done in triplicate and statistical analyses were done with the SAS software package. All data were normally distributed. For multiple mean comparisons, we conducted ANOVA analysis by the Student's t-test for single mean comparison. P values less than 0.05 were considered significant.

\section{Results}

In order to examine whether Kav001 also inhibits other cytokines besides TNF- $\alpha$, we made a relevant experiment via ELISA. As shown in Figure 1A-1C, co-treatment of LPS plus Kav001 (A-C, \#4) significantly reduces LPS-induced TNF- $\alpha$, IL-1 $\beta$, or IL-6 compared to the positive controls (A-C, \#2). This phenomenon for detection of TNF- $\alpha$ was also observed while co-treatment of LPS plus Kavain but was not detectable for IL- $1 \beta$ or IL- 6 production (1B\&C, \#3) As shown in Figure 1D,1B. Additionally, neither Kavain nor Kav001 was found to affect IL-4. Because our preliminary data indicate that Kav001 affects some cytokines in response to LPS, we were therefore interested in whether Kav001 is involved in other stimuli-mediated cytokines. As shown in Figure 1E \& 1G, Kav001 strongly reduced all three stimuli-induced cytokine productions, TNF- $\alpha$ (E, No.4), IL-1 $\beta$ (F, No.4), and IL-6 (G, No.4) compared to their positive control (No.2 of E, F, or G). This phenomenon for reduction of IL-1 $\beta$ (F, No.3) or IL-6 (G, No.3) was not observed with co-treatment of stimuli plus Kavain. Since it is known that caspase 1 expression is activated with TNF- $\alpha$, IL-1 beta, or IL-6 [12-14], we wanted to further test whether Kav001 inhibits caspase 1 caused by TNF- $\alpha$, IL-1 beta, or IL-6. As shown in Figure 1F \& 1H, Kav001 $(H, N o .6)$ reduced LPS-induced caspase 1 compared to the positive control (H, No.2).

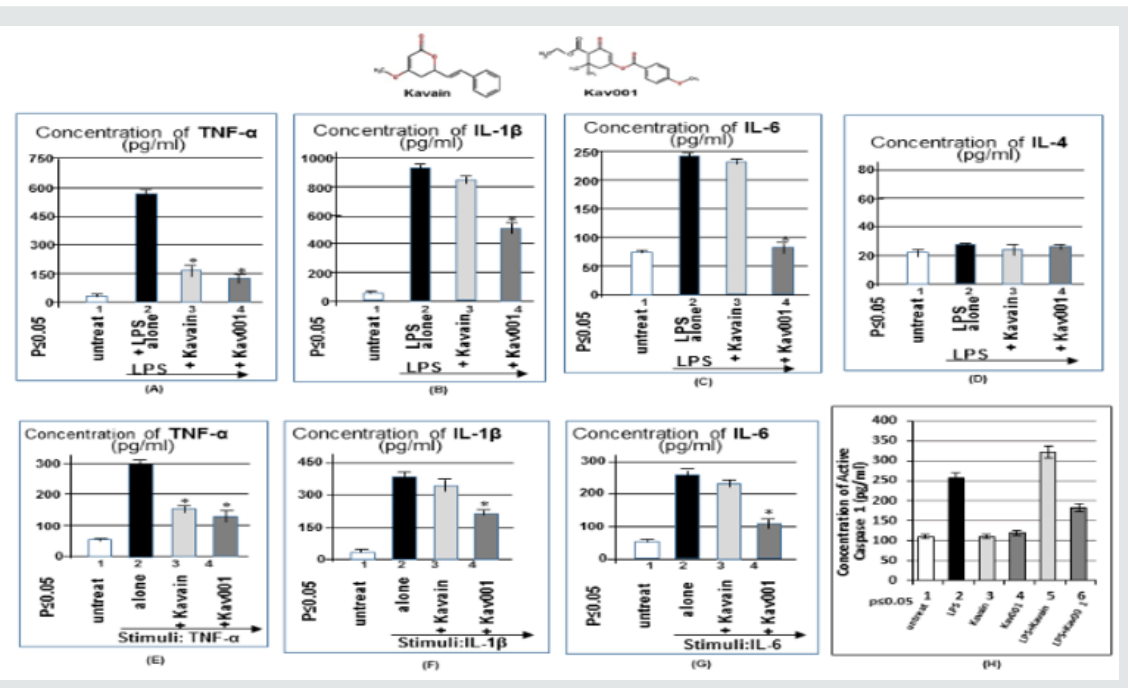

Figure 1: ELISA analysis. THP-1 cells were untreated as control (No.1, A-D) or treated with LPS alone $(0.1 \mu \mathrm{g} / \mathrm{ml}, \mathrm{No} .2$ of A-D) as positive control, or co-treated with LPS $(0.1 \mu \mathrm{g} / \mathrm{ml})$ plus Kavain $(100 \mu \mathrm{g} / \mathrm{ml}$, No.3 of A-D) or plus Kav001 $(100 \mu \mathrm{g} / \mathrm{ml}, \mathrm{No} .4$ of A-D) o/n. The medium from each group was detected by ELISA with the antibody against TNF- $\alpha$ (A), IL-1 $\beta$ (B), IL-6 (C), or IL-4 (D) and analyzed. THP-1 cells were untreated as control (No.1, E-G) or treated with 5ng/ml TNF- $\alpha$ (E, No.2-4), 10ng/ $\mathrm{ml}$ IL-1 $\beta$ (F, No.2-4), or 10ng/ml IL-6 (G, No.2-4), plus with $100 \mu \mathrm{g} / \mathrm{ml}$ of Kavain (E=G, No.3) or plus $100 \mu \mathrm{g} / \mathrm{ml} \mathrm{Kav001}$ (E-G, No. 4) for 2 hrs. Cells were then washed with $\mathrm{pBS}$ and cultured overnight. The treated medium from each group was measured

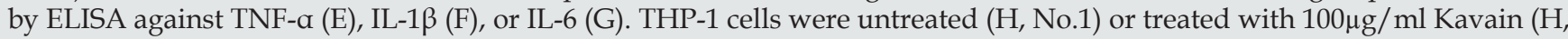
No.3),100 $\mu \mathrm{g} / \mathrm{ml} \mathrm{Kav001} \mathrm{(H,} \mathrm{No.4),} \mathrm{0.1 \mu g/ml} \mathrm{LPS} \mathrm{(H,} \mathrm{No.2)} \mathrm{ass} \mathrm{positive} \mathrm{control,} \mathrm{or} \mathrm{co-treated} \mathrm{with} \mathrm{LPS} \mathrm{plus} \mathrm{Kavain} \mathrm{(H,} \mathrm{No.5)}$ or plus Kav001 (H, No.6) o/n. The treated medium from each group was measured by ELISA against caspase 1. Note: The chemical structure of Kavain or Kav001 was attached above. 
As a control, Kavain did not reduce LPS-induced caspase 1, but instead increased its value (H, No.5). In order to analyze whether the treatment of Kav001 affects any kinases/protein upstream of caspase 1 in response to LPS, a kinase/protein array was performed. As shown in Figure 2B \& 2C Kav001 significantly reduced LPSinduced kinases such as Akt (Protein kinase B) at 17\%, ATM (Ataxia telangiectasia mutated) at $13 \%$, caspase 3 at $11.5 \%$, caspase 7 at 8.6\%, CHK1 (Checkpoint kinase 1) at 26.4\%, CHK2 (Checkpoint kinase 2) at $15.3 \%$, and ERK1/2 at $12.7 \% / 14.8 \%$, but does not affect p27, p53, or TAK1 (Transforming growth factor beta-activated kinase 1 (Figure 2A \& 2D). As it is known that subcutaneous injection of LPS in mice induces neutrophil infiltration of tissues $[15,16]$, we were therefore interested in whether Kav001 treatment is able to suppress LPS-induced neutrophil infiltration. As shown in Figure 3A \& 3D, indeed, subcutaneous treatment of 30mg/kg of Kav001 significantly reduced LPS-induced Neutrophil infiltration of tissues in mouse (DI,II, III) compared to the positive control (CI,II, III).

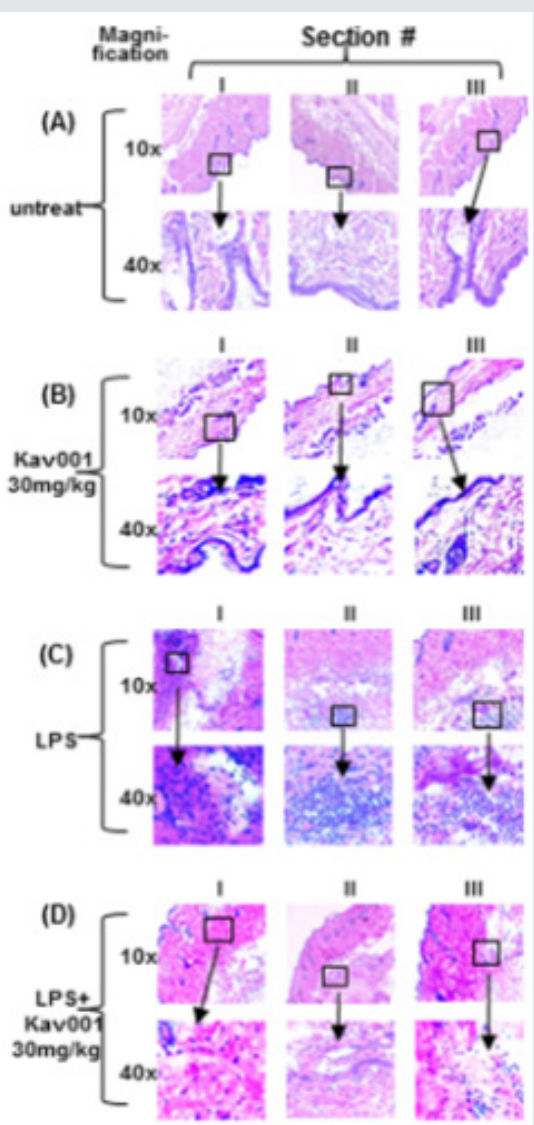

Figure 2: Kinase/Protein array. (A) The stamped identification number on the array. (B) THP-1 cells were untreated or treated with LPS alone or LPS+ Kav001 over night. The conditioned medium from each test group was assessed by protein assay and quantified by a Versace Imaging System. (C) Analysis of the intensity on the array filter. The cell intensity treated by LPS was assigned to $100 \%$ as base value and compared to the others. There ratio was calculated in this table.

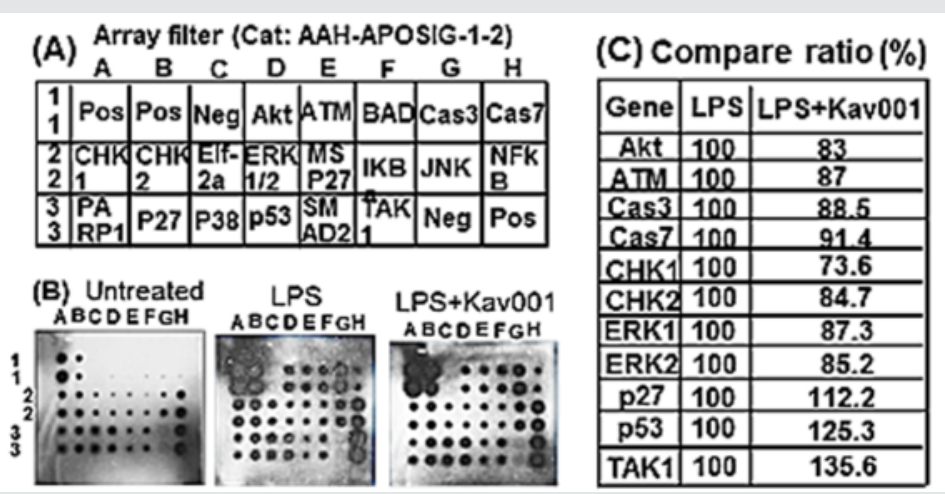

Figure 3: Neutrophil infiltration assay. Mice $(\mathrm{n}=3)$ were untreated (A) or oral gavage-treated with 30mg/kg of KaV001 alone (B) as negative control or treated by i.p. injection of LPS (1mg/ kg body) alone as the positive control (C) or co-treated with i.p. injection of LPS (1mg/ kg body) plus oral gavage of Kav001 (30mg/ kg) as test group (D) for $24 \mathrm{hrs}$. Skin tissues from each group were taken and fixed in $4 \%$ paraformaldehyde, then embedded in O.C.T. compound. Skin sections $(5 \mu \mathrm{m})$ were prepared, routinely stained with hematoxylin and eosin (H\&E) and imaged. 


\section{Discussion}

Kavain was shown to affect TNF- $\alpha$ transcriptional regulation [68]. Compared with Kavain, Kav001 not only inhibits LPS-induced TNF- $\alpha$, but also IL-1 $\beta$, IL-6, caspase 1 or Neutrophil infiltration. DMSO is known to be widely used as reagent solvent but sometimes causes a little toxicity to mammalian cells [17]. It is known that Kavain is dissolved in the higher concentration of DMSO ( $\geq 25 \%)$ while Kav001 is completely dissolved in water. This water-soluble property may be the reason why Kav001 prevent DMSO-mediated toxicity to cells with a longer period of efficacy. Our most recent data support this potential hypothesis [9]. Additionally, neutrophils are the innate immune response and thus, neutrophilic infiltration of tissues is involved in many inflammatory diseases [18,19]. In order to inhibit the neutrophilic infiltration from tissue injury (I/R injury, brain injury, ethanol feeding-induced liver injury), several inhibitors such as MgIG (Magnesium isoglycyrrhizinate), Clenbuterol, Phillyrin, or PAI-1 (Plasminogen activator inhibitor-1) have been widely studied $[20,21]$. Researcher groups have also suggested that these inhibitors suppress infiltration of neutrophils via regulation of factors such as MyD88 or p38 MAPK $[22,23]$. However, these studies of certain inhibitors leave several questions, such as whether there is a common key point between their pathways. Our data here also show that the treatment of Kav001 significantly reduces LPS-induced neutrophilic infiltration. Thus, Kav001 may be used for suppression of neutrophil infiltration induced by other stimuli or injuries. Since TNF- $\alpha$, IL-1 $\beta$, or IL- 6 was found here to be inhibited by Kav001, we assume that Kav001 may have treatment for inflammatory diseases caused by these cytokines (TNF- $\alpha$, IL-1 $\beta$, or IL-6). We will try to address this hypothesis for future research.

\section{References}

1. Schliack H (1997) Kavain in diseases of the aged. Hippokrates 38(1): 2630 .

2. Schmidt N, Ferger B (2001) Neuroprotective effects of (+/-)-kavain in the MPTP mouse model of Parkinson's disease. Synapse 40(1): 47-54.

3. Alshammari A, Patel J, Al Hashemi J, Cai B, Panek J, et al. (2017) Kava241 reduced periodontal destruction in a collagen antibody primed Porphyromonas gingivalis model of periodontitis. J Clin Periodontol 44(11): 1123-1132.

4. Yuan H, Gupte R, Zelkha S, Amar S (2011) Receptor activator of nuclear factor kappa B ligand antagonists inhibit tissue inflammation and bone loss in experimental periodontitis. J Clin Periodontol 38(1): 1029-1036.

5. Folmer F, Blasius R, Morceau F, Tabudravu J, Dicato M, et al. (2006) Inhibition of TNFalpha-induced activation of nuclear factor kappa B by kava (Piper methysticum) derivatives. Biochem Pharmacol 71(8): 12061218

6. Tang X, Amar S (2016) Kavain Involvement in LPS-Induced Signaling Pathways. J Cell Biochem 117(10): 2272-2280.

7. Pollastri MP, Whitty A, Merrill JC, Tang X, Ashton TD, et al. (2009) Identification and characterization of kava-derived compounds mediating TNF-alpha suppression. Chem Biol Drug Des 74(2): 121-128.
8. Tang X, Amar S (2016) Kavain Inhibition of LPS-Induced TNF- $\alpha$ via ERK/ LITAF. Toxicol Res (Camb) 5(1): 188-196.

9. Tang X, Alasiri M, Bamashmous A, Aljahdali B, Cao F, et al. (2018) The involvement of Kav001 in inhibition of LPS/P. gingivalis-induced. J Cell Biochem 119(7): 6072-6079.

10. Turbett GR, Sellner LN (1997) The use of optimal cutting temperature compound can inhibit amplification by polymerase chain reaction. Diagn Mol Pathol 6(5): 298-303.

11. Wang Q Jotwani R, Le J, Krauss JL, Potempa J, et al. (2014) Filifactor alocis infection and inflammatory responses in the mouse subcutaneous chamber model. Infect Immun 82(3): 1205-1212.

12. Jin H, Jin X, Cao B, Wang W (2017) Berberine affects osteosarcoma via downregulating the caspase-1/IL-1 $\beta$ signaling axis. Oncol Rep 37(2): 729-736.

13. Wang CC, Zhang M, Li H, Li XL, Yue LT, et al. (2017) Caspase-1 inhibitor regulates humoral responses in experimental autoimmune myasthenia gravis via IL-6- dependent inhibiton of STAT3. Neurosci Lett 656: 169176.

14. Madhu BP, Singh KP, Saminathan M, Singh R, Tiwari AK, et al. (2016) Correlation of inducible nitric oxide synthase (iNOS) inhibition with TNF- $\alpha$, caspase-1, FasL and TLR-3 in pathogenesis of rabies in mouse model. Virus Genes 52(1): 61-70.

15. Johnson JL, Hong H, Monfregola J, Catz SD (2011) Increased survival and reduced neutrophil infiltration of the liver in Rab27a- but not Munc134-deficient mice in lipopolysaccharide-induced systemic inflammation. Infect Immun 79(9): 3607-3618.

16. Amison RT, Arnold S, O’Shaughnessy BG, Cleary SJ, Ofoedu J, et al. (2017) Lipopolysaccharide (LPS) induced pulmonary neutrophil recruitment and platelet activation is mediated via the P2Y1 and P2Y14 receptors in mice. Pulm Pharmacol Ther 45: 62-68.

17. Galvao J, Davis B, Tilley M, Normando E, Duchen MR, et al. (2014) Unexpected low-dose toxicity of the universal solvent DMSO. FASEB J 28(3): 1317-1330.

18. Griffin ÉW, Yssel JD, O’Neill E, Ryan KJ, Boyle N, et al. (2018) The $\beta 2$ adrenoceptor agonist clenbuterol reduces the neuroinflammatory response, neutrophil infiltration and apoptosis following intra-striatal IL-1 $\beta$ administration to rats. Immunopharmacol Immunotoxicol 40(2): 99-106.

19. Kim YJ, Shin JM, Shin SH, Kim JH, Sohn KY, et al. (2017) 1-palmitoyl2-linoleoyl-3-acetyl-rac-glycerol ameliorates arthritic joints through reducing neutrophil infiltration mediated by IL-6/STAT3 and MIP-2 activation. Oncotarget 8(57): 96636-96648.

20.Zou X, Wang Y, Peng C, Wang B, Niu Z, et al. (2018) Magnesium isoglycyrrhizinate has hepatoprotective effects in an oxaliplatin-induced model of liver injury. Int J Mol Med 42: 2020-2030.

21. Chen B, Zhang Y, Chen L, Huang S, Li S, et al. (2013) Dose-effects of aorta-infused clenbuterol on spinal cord ischemia-reperfusion injury in rabbits. PLoS One 8: e84095.

22. Weil ZM, Karelina K, Gaier KR, Corrigan TE, Corrigan JD (2016) Juvenile Traumatic Brain Injury Increases Alcohol Consumption and Reward in Female Mice. J Neurotrauma 33(9): 895-903.

23. Hesketh EE, Czopek A, Clay M, Borthwick G, Ferenbach D, et al. (2014) Renal ischaemia reperfusion injury: a mouse model of injury and regeneration. J Vis Exp 88: 51816. 
(C) (i) This work is licensed under Creative

To Submit Your Article Click Here:

Submit Article

DOI: $10.32474 /$ LOJPCR.2019.01.000122

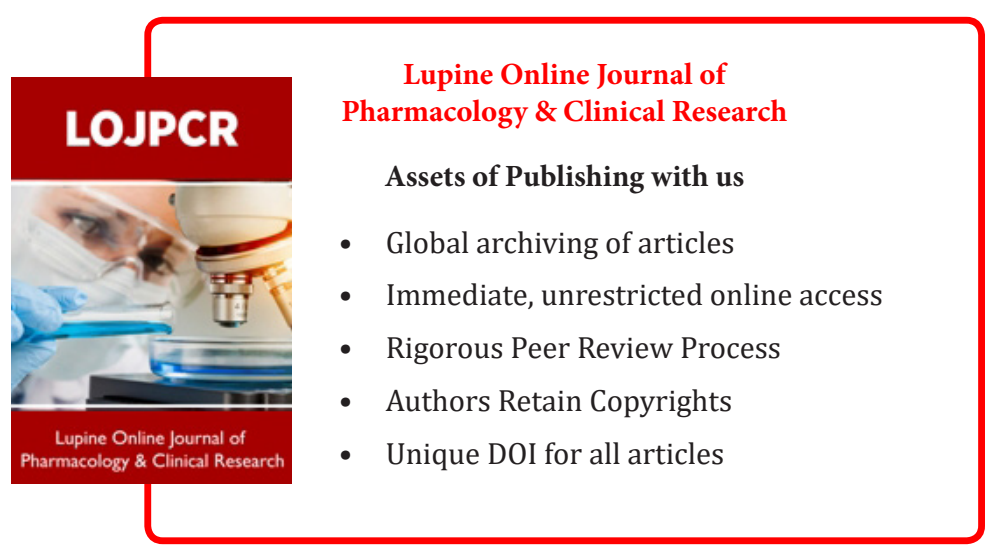

